Stochastic Processes and Operator Calculus on Quantum Groups 
Managing Editor:

M. HAZEWINKEL

Centre for Mathematics and Computer Science, Amsterdam, The Netherlands 


\section{Stochastic Processes and Operator Calculus on Quantum Groups}

by

Uwe Franz

Institut für Mathematik und Informatik,

Ernst-Moritz-Arndt-Universität Greifswald,

Greifswald, Germany

and

René Schott

Institut Elie Cartan and Loria,

Université Henri Poincaré-Nancy l,

Vandoeuvre-lès-Nancy,

France

Springer-Science+Business Media, B.V. 
A C.I.P. Catalogue record for this book is available from the Library of Congress.

ISBN 978-90-481-5290-2 ISBN 978-94-015-9277-2 (eBook)

DOI 10.1007/978-94-015-9277-2

Printed on acid-free paper

All Rights Reserved

(C) 1999 Springer Science+Business Media Dordrecht

Originally published by Kluwer Academic Publishers in 1999.

Softcover reprint of the hardcover 1st edition 1999

No part of the material protected by this copyright notice may be reproduced or utilized in any form or by any means, electronic or mechanical, including photocopying, recording or by any information storage and retrieval system, without written permission from the copyright owner. 


\section{Contents}

Preface vii

1 Introduction 1

2 Preliminaries on Lie groups 5

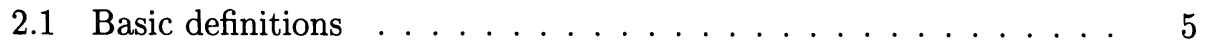

2.2 Examples . . . . . . . . . . . . . . . . . . 7

2.3 Dual representations . . . . . . . . . . . . . . . . . . 9

2.4 The splitting lemma . . . . . . . . . . . . . . . 15

2.5 The composition law . . . . . . . . . . . . 20

2.6 Matrix elements . . . . . . . . . . . . . . . . . 22

2.7 Stochastic processes on Lie groups . . . . . . . . . . . . 24

3 Hopf algebras, quantum groups and braided spaces $\quad 45$

3.1 Coalgebras, bialgebras, and Hopf algebras . . . . . . . . . 46

3.2 Examples of bialgebras and Hopf algebras . . . . . . . . . . 50

3.3 Dual representations for quantum groups . . . . . . . . . 58

3.4 A composition law for quantum groups . . . . . . . . . . . 68

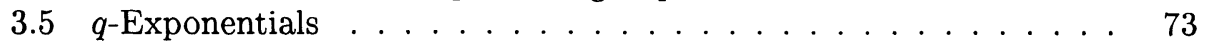

3.6 Matrix elements . . . . . . . . . . . . . . . 78

3.7 Braided tensor categories . . . . . . . . . . . . . 81

3.8 Braided bialgebras and braided Hopf algebras . . . . . . . . . 83

3.9 Examples of braided bialgebras and braided Hopf algebras . . . . 86

3.10 Braided spaces . . . . . . . . . . . . . . . . . . . 88

3.11 Compact quantum groups $\ldots \ldots \ldots \ldots \ldots$

4 Stochastic processes on quantum groups 93

4.1 Quantum probability . . . . . . . . . . . . . . . 93

4.2 Independence . . . . . . . . . . . . . . . . . . . . 94

4.3 Lévy processes on bialgebras . . . . . . . . . . . . . . . 96

4.4 Realisation of Lévy processes on Fock spaces . . . . . . . . . 98

4.5 Realisation of Lévy processes by an inductive limit . . . . . . . . 102

4.6 Multiplicative stochastic integrals . . . . . . . . . . . . 105

4.7 Feynman-Kac formula . . . . . . . . . . . . . . . 106

4.8 Time-reversal, duality, and R-matrices . . . . . . . . . . . . 109 
5 Markov structure of quantum Lévy processes 119

5.1 Classical versions of quantum Lévy processes . . . . . . . . 120

5.2 Examples of classical versions of Lévy processes on $\mathbb{C}_{q}\left\langle a, a^{*}\right\rangle \ldots 125$

5.3 Examples of classical versions of Lévy processes on $U_{q}(s l(2)) \ldots 131$

5.4 Lévy processes on $U_{q}(a f f(1)) \ldots \ldots \ldots \ldots \ldots$

6 Diffusions on braided spaces 139

6.1 A construction of (pseudo-) diffusions on braided spaces . . . . . 140

6.2 Appell systems . . . . . . . . . . . . . . 146

6.3 Densities . . . . . . . . . . . . . . . . . . . 150

7 Evolution equations and Lévy processes on quantum groups 153

7.1 Appell systems . . . . . . . . . . . . . . . . . 153

7.2 Wigner-type densities $\ldots \ldots \ldots \ldots \ldots \ldots \ldots$

8 Gauss laws in the sense of Bernstein on quantum groups $\quad 161$

8.1 Gaussian functionals in the sense of Bernstein . . . . . . . . 161

8.2 Uniqueness of embedding . . . . . . . . . . . . . 171

8.3 Gaussian semi-groups in the sense of Bernstein . . . . . . . 175

9 Phase retrieval for probability distributions on quantum groups $\begin{array}{lr}\text { and braided groups } & 183\end{array}$

9.1 Classical simply connected nilpotent Lie groups . . . . . . . . . 184

9.2 The phase problem on the braided line . . . . . . . . . . 185

9.3 The phase problem on nilpotent quantum or braided groups . . . 185

9.4 On the braided plane . . . . . . . . . . . . . . . 187

9.5 On the braided Heisenberg-Weyl group . . . . . . . . . . 187

10 Limit theorems on quantum groups $\quad 189$

10.1 Analogues of the law of large numbers and the central limit theorem 189

10.2 A mixed quantum-classical central limit theorem . . . . . . . . . 191

10.3 Convergence to the Haar measure on compact quantum groups . . 198

$10.4 q$-central limit theorem for $U_{q}(s u(2)) \ldots \ldots \ldots \ldots$. . . . . 198

10.5 Domains of attraction for $q$-transformed random variables . . . . 206

$\begin{array}{ll}\text { Bibliography } & 215\end{array}$

$\begin{array}{ll}\text { Index } & 225\end{array}$ 


\section{Preface}

Quantum groups have been investigated rather deeply in mathematical physics over the last decade. Among the most prominent contributions in this area let us mention the works of V.G. Drinfeld, S.L. Woronowicz, S. Majid. Probability theory on quantum groups has developed in several directions (see works of P. Biane, R.L. Hudson and K.R. Partasarathy, P.A. Meyer, M. Schürmann, D. Voiculescu). The aim of this book is to present several new aspects related to quantum groups: operator calculus, dual representations, stochastic processes and diffusions, Appell polynomials and systems in connection with evolution equations. Much of the material is scattered throughout available literature, however, we have nowhere found in accessible form all of this material collected. The presentation of representation theory in connection with Appell systems is original with the authors. Stochastic processes (example: Brownian motion, diffusion processes, Lévy processes) are investigated and several examples are presented.

As a text the work is intended to be accessible to graduate students and researchers not specialised in quantum probability.

We would like to acknowledge our colleagues P. Feinsilver, R. Lenzceswki, D. Neuenschwander, and M. Schürmann for allowing us to use material which has been the object of joint works. We would like as well to acknowledge our colleagues from Clausthal (Arnold Sommerfeld Institute), Greifswald (Institut für Mathematik und Informatik), Nancy (Institut Elie Cartan and LORIA), Strasbourg (IRMA) for numerous discussions on the topic of this book. Finally we express gratitude to our families and to all our friends. Their patience and encouragement made this project possible. 